\title{
Correction to: In Vitro-In Vivo Dose Response of Ursolic Acid, Sulforaphane, PEITC, and Curcumin in Cancer Prevention
}

\author{
Christina N. Ramirez, ${ }^{1,2}$ Wenji Li, ${ }^{1,3}$ Chengyue Zhang, ${ }^{1,3,4}$ Renyi Wu, ${ }^{1,3}$ Shan Su, ${ }^{1,3}$ Chao Wang, ${ }^{1,3}$ Linbo Gao, ${ }^{1,3}$ \\ Ran Yin, ${ }^{1,3}$ and Ah-Ng Tony Kong ${ }^{1,3,4,5,6}$
}

\section{Published online 6 February 2018}

Correction to: AAPS J

https://doi.org/10.1208/s12248-017-0177-2

The citation of the author name "Ah-Ng Tony Kong" in PubMed is not the author's preference. Instead of "Kong AT", the author prefers "Kong AN".

The online version of the original article can be found at https://doi.org/10.1208/s12248-017-0177-2

${ }^{1}$ Center for Phytochemicals Epigenome Studies, Ernest Mario School of Pharmacy, Rutgers, The State University of New Jersey, Piscataway, New Jersey 08854, USA.

${ }^{2}$ Cellular and Molecular Pharmacology Program, Rutgers Robert Wood Johnson Medical School, Piscataway, New Jersey 08854, USA.

${ }^{3}$ Department of Pharmaceutics, Ernest Mario School of Pharmacy, Rutgers, The State University of New Jersey, Piscataway, New Jersey 08854, USA.

${ }^{4}$ Graduate Program in Pharmaceutical Sciences, Department of Pharmaceutics, Ernest Mario School of Pharmacy, Rutgers, The State University of New Jersey, Piscataway, New Jersey 08854, USA.

${ }^{5}$ Ernest Mario School of Pharmacy, Room 228, Rutgers, The State University of New Jersey, 160 Frelinghuysen Road, Piscataway, New Jersey 08854, USA.

${ }^{6}$ To whom correspondence should be addressed. (e-mail: kongt@pharmacy.rutgers.edu) 